\title{
Self-inflicted transorbital brain injury by chopsticks in a patient with acute psychosis
}

YC Lee ${ }^{1 \text { * }}$ MB, ChB, MRCP, HH Kwan², MB, BS, MRCP, T Wong1', MB, ChB, FRCR, NY Pan'1 FRCR, FHKAM (Radiology), HY Lai' ${ }^{1}$, FRCR, FHKAM (Radiology), KF Ma', FRCR, FHKAM (Radiology)

\author{
${ }^{1}$ Department of Radiology \\ 2 Division of Neurology, Department of Medicine \\ Princess Margaret Hospital, Laichikok, Hong Kong
}

*Corresponding author: lyc713@ha.org.hk

All authors contributed equally to this case report.

Hong Kong Med J 2017;23:313-4

DOI: 10.12809/hkmj154644

\section{Case report}

A 56-year-old man was admitted from the emergency department with acute onset of psychotic symptoms and suicidal ideation in January 2015. While under observation in the emergency medical ward, he self-inflicted orbital injuries with two plastic chopsticks. The chopsticks were pulled out by the patient. Further injury was immediately stopped by the attending physician. Urgent computed tomographic (CT) brain showed two almostparallel haemorrhagic penetration tracts extending posterosuperiorly from bilateral inferior frontal lobes (Fig a, b) to involve the caudate and lentiform nuclei (Fig c), barely missing the internal capsules.
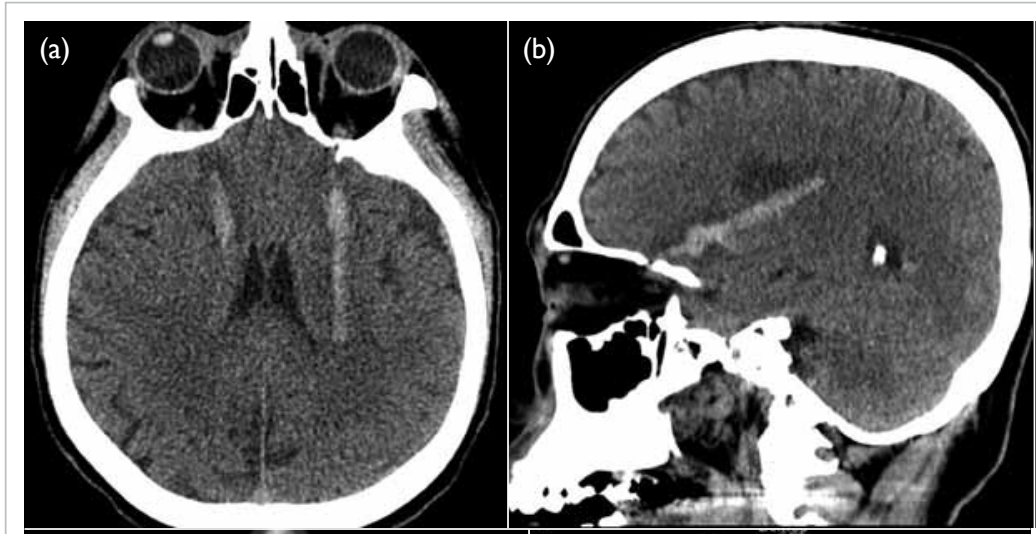

(c)
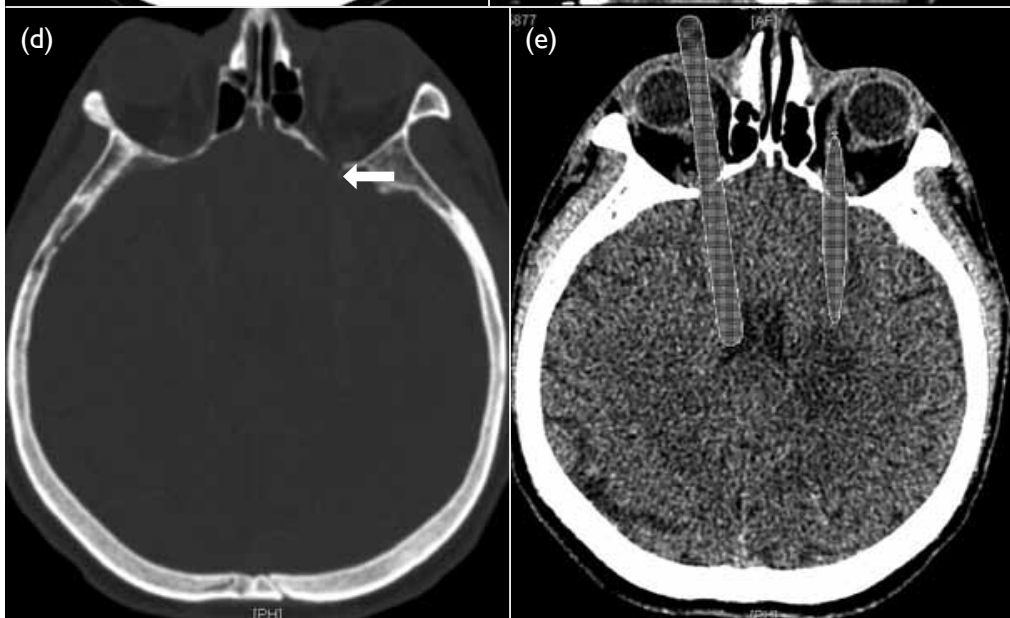

FIG. Computed tomographic images of the patient

(a) Axial and (b) sagittal images showing bilateral near-parallel haemorrhagic penetration tracts extending from bilateral inferior frontal lobes posterosuperiorly to the basal ganglia. (c) An axial image showing right caudate and left lentiform nuclei involvement. (d) A reformatted image showing a tiny bone fragment (arrow) superior to the left orbital roof, indicating fracture. (e) Chopstick trajectories were recreated by extrapolating the course of the linear haematomas 
Reformatted images revealed defects in both orbital roofs (Fig d), indicating fractures. The trajectories of penetration were recreated by extrapolating the linear haematomas on a workstation (Fig e). No retained foreign body was detected. Subsequent digital subtraction angiogram and CT angiogram did not demonstrate any vascular injury. The patient was clinically and neurologically stable throughout the admission, and was managed conservatively without any neurosurgical intervention. The patient's psychiatric symptoms gradually subsided after starting antipsychotic medication and he was discharged without significant neurological deficit. Only mild cogwheel rigidity in the upper limbs was noted during neurological follow-up.

\section{Discussion}

There have been a few case reports of self-inflicted penetrating brain injury in patients with psychiatric symptoms. The methods of injury were often bizarre and associated with a high mortality rate. ${ }^{1}$

Transorbital penetrating brain injury is rare but accounts for nearly one quarter of penetrating head injuries in adults, and one half of those in children. ${ }^{2}$ Penetrating objects such as wood, ${ }^{3}$ chopsticks, ${ }^{4}$ pens and pencils ${ }^{2}$ have been commonly implicated.

The orbit is shaped like a horizontal pyramid. This special anatomy often directs the penetrating object towards the apex and into the brain through three common sites. ${ }^{2}$ The most common pathway is through the orbital roof, as in our case. This causes frontal lobe contusion or laceration and may injure the anterior cerebral arteries. The next most common path is via the superior orbital fissure in which the cavernous sinus, brainstem, and cranial nerves can be injured. Penetration through the optical canal is rare. ${ }^{5}$ The penetrating object is directed into the suprasellar cistern near the optic nerve and internal carotid artery. ${ }^{6,7}$

Neurological signs and symptoms may initially be absent even when both intracranial haematoma and foreign body are present, but a patient who is conscious on presentation can deteriorate rapidly and therefore neurological surveillance is needed. ${ }^{3,7}$ A high degree of suspicion is required especially when handling psychiatric patients because injuries can be subtle and initially occult. ${ }^{7}$

Of note, $\mathrm{CT}$ is the initial investigation to delineate the extent of injury, determine the presence of retained foreign bodies, and plan subsequent surgery. Digital subtraction angiogram or CT angiogram is helpful when there is a suggestion of vascular injury, either by the location and trajectory of the foreign body or by evidence of haematoma on initial CT scan. ${ }^{2}$

The patient in this case report was fortunate because the trajectories of both penetration tracts narrowly missed the internal capsules, preventing major neurological deficit. Chung et $\mathrm{al}^{8}$ reported that small haematomas involving caudate or lentiform nuclei cause only mild-to-moderate or even no motor and sensory deficit, as in our case. Injury to the caudate and lentiform nuclei, however, may explain the late-onset cogwheel rigidity of upper limbs in our patient. This case also illustrates that the apparent severity of cerebral trauma on CT may not correlate with a patient's initial degree of neurological deficit. Close follow-up in an out-patient clinic is required to detect any subtle late-onset neurological signs.

\section{Declaration}

All authors have disclosed no conflicts of interest.

\section{References}

1. Greene KA, Dickman CA, Smith KA, Kinder EJ, Zabramski JM. Self-inflicted orbital and intracranial injury with a retained foreign body, associated with psychotic depression: case report and review. Surg Neurol 1993;40:499-503.

2. Schreckinger M, Orringer D, Thompson BG, La Marca F, Sagher O. Transorbital penetrating injury: case series, review of the literature, and proposed management algorithm. J Neurosurg 2011;114:53-61.

3. Borkar SA, Garg K, Garg M, Sharma BS. Transorbital penetrating cerebral injury caused by a wooden stick: surgical nuances for removal of a foreign body lodged in cavernous sinus. Childs Nerv Syst 2014;30:1441-4.

4. Mitilian D, Charon B, Brunelle F, Di Rocco F. Removal of a chopstick out of the cavernous sinus, pons, and cerebellar vermis through the superior orbital fissure. Acta Neurochir (Wien) 2009;151:1295-7.

5. Matsumoto S, Hasuo K, Mizushima A, et al. Intracranial penetrating injuries via the optic canal. AJNR Am J Neuroradiol 1998;19:1163-5.

6. Di Roio C, Jourdan C, Mottolese C, Convert J, Artru F. Craniocerebral injury resulting from transorbital stick penetration in children. Childs Nerv Syst 2000;16:503-6.

7. Turbin RE, Maxwell DN, Langer PD, et al. Patterns of transorbital intracranial injury: a review and comparison of occult and non-occult cases. Surv Ophthalmol 2006;51:449-60.

8. Chung CS, Caplan LR, Yamamoto Y, et al. Striatocapsular haemorrhage. Brain 2000;123(Pt 9):1850-62. 